\title{
Is pure autonomic failure an early marker for Parkinson disease, dementia with Lewy bodies, and multiple system atrophy? And other updates on recent autonomic research
}

\author{
Srikanth Muppidi ${ }^{1} \cdot$ Mitchell G. Miglis $^{1}$
}

Received: 13 February 2017/Accepted: 14 February 2017/Published online: 2 March 2017

(C) Springer-Verlag Berlin Heidelberg 2017

\section{Is pure autonomic failure really pure?}

Pure autonomic failure (PAF) is a disorder of $\alpha$-synuclein deposition that results in neurogenic orthostatic hypotension $(\mathrm{OH})$ and other features of autonomic failure, without signs of central nervous system (CNS) involvement. If patients are followed for a sufficient period, some may eventually develop Parkinson disease (PD), multiple system atrophy (MSA), or dementia with Lewy bodies (DLB). Determining which patients will go on to convert to a CNS synucleinopathy and which will remain as PAF has been, so far, a prognostic dilemma for medical providers. Is PAF purely peripheral and restricted to the autonomic nerves, or does it presage a more widespread CNS disorder?

A recent pivotal paper published in the Annals of Neurology by members of the U.S. Autonomic Disorders Consortium reports the results of the natural history of pure autonomic failure, enrolling patients with PAF and followed annually for a 4-year period [3]. All subjects underwent extensive clinical and autonomic testing, including cognitive screening, olfaction, and supine, and upright serum norepinephrine levels.

This multi-center study performed at five U.S. sites enrolled 75 patients with PAF, the largest cohort reported to date. The majority of them were male $(70 \%)$, and the mean duration of disease since onset of autonomic symptoms was 5 years. Ninety-five percent of patients reported orthostatic intolerance, $58 \%$ constipation, $50 \%$ urinary symptoms, $44 \%$ abnormalities of sweating, and $65 \%$ of

Srikanth Muppidi

muppidis@stanford.edu

1 Department of Neurology, Stanford Medical Center, 213 Quarry Road, 2nd Floor, Palo Alto, CA 94304, USA men reported erectile dysfunction. All patients had evidence of $\mathrm{OH}$ on tilt testing, and all patients had an absent phase IV overshoot on Valsalva maneuver, indicative of neurogenic $\mathrm{OH}(\mathrm{nOH})$. Seventy-one percent had reduced heart rate variability with deep breathing and 54\% had reduced serum norepinephrine levels on upright tilt. The prevalence of probable rapid eye movement (REM) sleep behavior disorder (RBD) by questionnaires was $72 \%$, raising the possibility of brainstem involvement. Interestingly, a similar prevalence of polysomnography-confirmed RBD (63\%) was documented in a small cohort of PAF patients [4]. Olfactory function was significantly impaired in $73 \%$ of the natural history cohort, further indicating CNS involvement.

At 1-year follow up, $15 \%$ of patients converted to a CNS synucleinopathy (two PD, four DLB, and five MSA). The conversion rates of the remaining patients at years two, three, and four were 13,13 , and $27 \%$, respectively. Predictors of phenoconversion to PD/DLB included impaired olfactory function with probable RBD, and phenoconverstion to MSA included preserved olfaction with probable RBD. Subtle motor deficits were noted in $88 \%$ of patients that converted to a CNS synucleinopathy. This study supports the notion that $\alpha$-synuclein spreads in a prion-like fashion through adjacent cells. However, not all patients with a PAF phenotype may be associated with $\alpha$-synuclein spread, as described in a case-report in this issue of Clinical Autonomic Research [2].

The Natural History Study of Synucleinopathies continues on its second iteration, as a multi-center, National Institutes of Health (NIH)-sponsored study hoping to expand these results. Several sites in North America, South America, Europe, and Asia participating in this study are currently enrolling patients with PAF, MSA and idiopathic RBD in order to document the natural history of these 
disorders and clarify which factors predict phenoconversion to PD, DLB, or MSA. Long-term studies such as this will be essential to help clarify the natural progression of these diseases so that we may better inform our patients regarding their prognosis.

\section{Let's sit down and think about this}

Neurogenic $\mathrm{OH}(\mathrm{nOH})$ is a well-known non-motor feature of Parkinson disease (PD). Bedside orthostatic blood pressure measurements in the supine and standing positions can identify patients with $\mathrm{nOH}$, although, regrettably, this is not routinely performed by most practitioners. Instead most physicians rely on patients to report orthostatic symptoms before investigating further.

However, not all patients with PD and nOH may present with typical symptoms of orthostatic intolerance such as lightheadedness or presyncope. It has been well described that some patients may manifest with fatigue, trouble concentrating, or even cognitive impairment that is truly orthostatic in nature. An effort to understand the role of decreased cerebral perfusion as a result of $\mathrm{nOH}$ and it's relationship to cognitive function in PD patients was recently published in Neurology [1]. The authors recruited patients with $\mathrm{PD}$, either without or with known orthostatic hypotension $(\mathrm{PDOH})$, and controls without $\mathrm{PD}$. All patients underwent various standardized tests of cognitive function in both the supine and head-up tilt positions.

Not surprisingly, all PD patients had executive dysfunction in the supine position when compared to controls. However, the PDOH group had more cognitive impairment in the head-up position. This finding was not observed in patients without $\mathrm{OH}$. This study supports the hypothesis that $\mathrm{OH}$ contributes to cognitive impairment in the upright position, even though patients might not report the classic symptoms of dizziness or lightheadedness.

The relationship between cognition and orthostatic hypotension is also the topic of a recent review article published in the Journal of Neurology, Neurosurgery, and Psychiatry [6]. The authors assert that there is clear relationship between $\mathrm{OH}$ and cognitive dysfunction in a $\alpha$ synucleinopathies and propose multiple potential mechanisms, including cerebral hypoperfusion leading to cognitive impairment, diffuse $\alpha$-synuclein neuropathology causing both cognitive impairment and $\mathrm{OH}$, and a combination of these two underlying etiologies. While we await further studies to elaborate on these hypotheses, the association between posture and cognitive change in this population deserves greater attention, and it behooves all neurologists to sit down and discuss these findings with their PD patients.

\section{Autonomic dysfunction and fitness in patients with recently diagnosed diabetes}

Cardiac autonomic dysfunction is one of the more serious neurological complications of long standing diabetes. Cardiac autonomic dysfunction with reduced heart rate variability (HRV) may contribute to reduced cardiorespiratory fitness in patients with diabetes, and thus increases morbidity and mortality risk. While these complications are more common in chronic diabetes, the association is not as well described in newly-diagnosed diabetes. In a recent research article published in Diabetes Care [5], investigators recruited 163 recently diagnosed patients with type-1 diabetes mellitus, 188 patients with type- 2 diabetes mellitus, and 40 age-matched controls for each group. All study participants underwent HRV assessment and spiroergometry to assess autonomic function and cardiorespiratory fitness during a hyperinsulenemic-euglycemic clamp.

As expected, patients with type-2 diabetes were older and had a higher body mass index when compared to patients with type-1 diabetes. Cardiorespiratory fitness was lower in type- 2 patients but not in type-1 diabetes patients, when compared to age-matched controls. HRV measures were reduced in both type-1 and type- 2 diabetes groups, but the association between diminished HRV measures and poor cardiorespiratory fitness was significant only in the type-1 group. These findings suggest that we should continue to recommend a robust exercise program in recently diagnosed patients with diabetes, as this may improve both cardiovascular fitness and HRV. As the authors note, we still do not know the temporal relationship between diminished HRV and cardiorespiratory fitness in patients with diabetes. However, as any autonomic specialist can attest, exercise is an integral part of most treatment plans, and in this particular population, may be just what the doctor ordered.

\section{Compliance with ethical standards}

Conflict of interest None.

\section{References}

1. Centi J, Freeman R, Gibbons $\mathrm{CH}$, Neargarder S, Canova AO, Cronin-Golomb A (2017) Effects of orthostatic hypotension on cognition in Parkinson disease. Neurology 88:17-24

2. Isonaka R, Holmes C, Cook GA, Sullivan P, Sharabi Y, Goldstein DS (2017) Pure autonomic failure without synucleinopathy. Clin Auton Res. doi:10.1007/s10286-017-0404-z

3. Kaufmann H, Norcliffe-Kaufmann L, Palma JA, Biaggioni I, Low PA, Singer W, Goldstein DS, Peltier AC, Shibao CA, Gibbons CH, Freeman R, Robertson D, Autonomic Disorders C (2017) The natural history of pure autonomic failure: a United States 
prospective cohort. Ann Neurol 81(2):287-297. doi:10.1002/ana. 24877

4. Miglis MG, Muppidi S, During E, Jaradeh S (2017) A case series of REM sleep behavior disorder in pure autonomic failure. Clin Auton Res 27:41-44

5. Rohling M, Strom A, Bonhof G, Puttgen S, Bodis K, Mussig K, Szendrodi J, Markgraf D, Lehr S, Roden M, Ziegler D, German Diabetes Study Group (2017) Differential patterns of impaired cardiorespiratory fitness and cardiac autonomic dysfunction in recently diagnosed Type 1 and Type 2 diabetes. Diabetes Care 40:246-252

6. Udow SJ, Robertson AD, MacIntosh BJ, Espay AJ, Rowe JB, Lang $\mathrm{AE}$, Masellis M (2016) 'Under pressure': is there a link between orthostatic hypotension and cognitive impairment in alpha-synucleinopathies? J Neurol Neurosurg Psychiatry 87:1311-1321 